\title{
ANATOMIA DA ARTÉRIA LINGUAL PROFUNDA EM Sus scrofa domestica, LINNAEUS, 1758
}

\author{
Jussara Rocha Ferreira ${ }^{1}$, Edwar Alves Carvalho², Waldemar de Souza ${ }^{2}$, Bruno Farah Alvarenga ${ }^{2}$, \\ BRUNO FERREIRA RODRIGUES ${ }^{2}$
}

1 - Professora doutora, UnB, Departamento de morfologia, área de anatomia - jussararocha@unb.br

2 - UnB, Departamento de morfologia, área de anatomia

RESUMO

\begin{abstract}
Foram estudadas as artérias da língua de Sus scrofa domestica (Linnaeus, 1758) de 20 animais cujos órgãos foram coletados a fresco e resfriados $\left(4^{\circ} \mathrm{C}\right)$ para transporte. Os vasos, em 15 espécimes, foram perfundidos com água $\left(37^{\circ} \mathrm{C}\right)$, injetados com látex, fixados em formaldeído (7\%) e dissecados sob lupa. Injetaram-se 5 peças com ar e acetona PA, depois ar novamente, por fim, solução de acetato de vinil corado. O material foi colocado em água por $24 \mathrm{~h}$ e, após, mergulhado em solução de ácido sulfúrico a $30 \%$ por duas semanas para obtenção de moldes do sistema arterial. As artérias forneceram ramos dorsais, ventrais e
\end{abstract}

colaterais como se segue: ramos dorsais na raiz da língua, três ramos $(50 \%)$, quatro ramos $(46,6 \%)$, cinco ramos $(3,4 \%)$; arranjos de ramos dorsais e ventrais no corpo da língua, um ventral e dois dorsais $(46,6 \%)$, um ventral e três dorsais $(23,3 \%)$, um ventral e um dorsal $(23,3 \%)$, um ventral e quatro dorsais $(3,4 \%)$ e um ventral $(3,4 \%)$. No ápice lingual a artéria seguiu paralela, entre os antímeros, encurvou-se medialmente, e se uniu à artéria contralateral estabelecendo uma anastomose por inosculação, formando ilhas arteriais, das quais partiram ramos radiais para o ápice da língua.

PALAVRAS-CHAVE: artérias linguais; língua; suínos; Sus scrofa domestica.

ABSTRACT

\section{ANATOMY OF THE DEEP LINGUAL ARTERY IN Sus scrofa domestica, Linnaeus,} 1758

We studied the arteries of the tongue of Sus scrofa doméstica (Linnaeus, 1758) from 20 animals whose tongues were slaughtered while fresh and kept cooled $\left(4^{\circ} \mathrm{C}\right)$ for transportation. The blood vessels of 15 animals were flowed with water $\left(37^{\circ} \mathrm{C}\right)$, injected with stained latex, fixated with phormaldeid and dissected with magnifying glass. We injected 5 tongues with air and acetone PA; later, we injected again with air and at last with vinyl stained acetate. The material was put in water for $24 \mathrm{~h}$ and, after this, submerged in sulfuric acid 30\% for two weeks to reveal the arterial system mold. The arteries provided dorsal, ventral and collateral branches in the following way: dorsal branches at the tongue root three branches $(50 \%)$, four branches $(46.6 \%)$, five branches (3.4\%); arrangements of the following dorsal and ventral branches at the tongue body - one ventral and two dorsal (46.6\%), one ventral and three dorsal $(23.3 \%)$, one ventral and one dorsal $(23.3 \%)$, one ventral and four dorsal $(3.4 \%)$ and one ventral branch (3.4\%). At the lingual apex, the artery was parallel between antimers, made a medial curve, and reached the counter-lateral artery, establishing anastomoses by inosculation, forming arterial islands from which radial branches to the tip of the tongue were originated.

KEYWORDS: lingual arteries; pigs; Sus scrofa domestica; swine; tongue. 


\section{INTRODUÇÃO}

A língua é um órgão formado pela junção de cinco elementos derivados da embriologia e que, em alguns animais, tem uma função importante na apreensão dos alimentos. SONNTAG (1920) considerava que as estruturas linguais variavam nos diferentes animais devido às diversidades de forma e função desse órgão muscular envolto por membrana mucosa e ricamente vascularizado. Em razão de sua especialização, nas diversas ordens de vertebrados, contempla músculos que podem prolongar-se até o exterior da cavidade oral, além de ser revestida por uma membrana mucosa, com papilas mecânicas e gustativas distribuídas no dorso do órgão (SONNTAG, 1920; SONNTAG, 1924; WATANABE \& KÖNIG, 1976; SCALZI, 1967; CHAMORRO et al., 1986). A mucosa lingual pode exibir rica rede capilar em primatas (MACHIDA et al.,1967) e nos animais domésticos (FRANDSON, 1979). Na parte ventral do órgão, a membrana mucosa forma uma prega medial, o frênulo lingual, assentado no assoalho da boca (WEICHERT, 1966).

A língua dos vertebrados recebe suprimento sanguíneo oriundo das artérias linguais e sublinguais, sendo que a artéria lingual, em ungulados, pode originar-se tanto da artéria carótida comum como da artéria carótida externa (LESBRE, 1923; DULZETTO, 1968; GETTY, 1981; SCHALLER, 1999; BERNARDINO JÚNIOR et al., 2003; SILVA et. al., 2003; FERREIRA et al., 2009).

As artérias linguais são vasos calibrosos que formam grande número de anastomoses arteriovenosas na mucosa lingual (RUBAY, 1950; PEREZ, 1956; DELLMANN \& BROWN, 1982; SAPPEY, 1894). Essas anastomoses foram também descritas na língua de ovinos e caprinos ocorrendo entre as camadas epitelial e muscular (PRICHARD \& DANIEL, 1954).

Tais artérias podem surgir como ramos colaterais da artéria maxilar em alguns ungulados (PEREZ, 1956; SCHWARZE \& SCHRÖDER, 1970), enquanto que em equinos e suínos podem ser originárias da artéria carótida externa a partir do denominado tronco línguo-facial (MONGIARDINO, 1905; MONTANÉ et al., 1949; GETTY, 1981; MUNIZ et al., 2006; MOTHEO et al., 2007). Em carnívoros, GETTY (1981) considerou que a artéria lingual pode eventualmente ser ramo da artéria maxilar.

Com relação à topografia dos vasos na língua, após penetrarem na musculatura extrínseca, as artérias ascendem rostralmente em direção ao ápice (GETTY, 1981). TAYLOR (1955) esquematizou uma artéria sublingual na borda ventro-lateral do músculo milohióide e uma artéria lingual na borda ventro-lateral dos músculos intrínsecos próxima ao septo lingual em animais domésticos. Na NOMINA ANATÔMICA VETERINÁRIA (2005), encontramse as denominações: artéria lingual, sublingual e artéria profunda da língua.

No que se refere à origem e distribuição dos ramos arteriais para a musculatura extrínseca e intrínseca da língua, a literatura é pouco esclarecedora, por isto a decisão de estudarmos os ramos da artéria lingual que irrigam a língua de Sus scrofa doméstica (Linnaeus, 1758).

Com este trabalho objetivou-se descrever a distribuição da artéria lingual na musculatura e demais tecidos da língua em suínos.

\section{MATERIAL E MÉTODOS}

Utilizamos 20 línguas de suínos adultos Sus scrofa domestica (Linnaeus, 1758), de ambos os sexos abatidos em frigorífico na região metropolitana de Goiânia, GO, com idade de seis a dez meses. O material foi coletado à fresco, resfriado em caixas isolantes com gelo e transportado até o laboratório. Após o transporte, os vasos foram perfundidos com água aquecida $\left(37^{\circ} \mathrm{C}\right)$. Látex corado com pigmento específico foi injetado em 15 línguas (30 antímeros). O material foi fixado em solução aquosa de formaldeído $7 \%$ e dissecado sob lupa (RANSOR II-20). A dissecação objetivou à identificação da artéria profunda da língua e à sistematização de suas subdivisões no parênquima lingual.

Com a finalidade de melhor elucidar a ramificação da artéria profunda da língua, em cinco peças, pela mesma via citada acima, o sistema de vasos da cabeça foi injetado com ar e acetona PA, depois ar novamente, e, por fim, solução de acetato de vinil corado com corante específico. O material foi então colocado em água por 24 horas sendo em seguida mergulhado em solução de ácido sulfúrico a $30 \%$ por um período de duas semanas para fins de obtenção dos moldes do sistema arterial. 
Neste estudo observaram-se os arranjos arteriais dos vasos dependentes da artéria profunda da língua de Sus Scrofa domestica (Linnaes, 1758). Para tanto, abordou-se a artéria lingual entre o osso hióide e a borda ventral do músculo hioglosso em 30 antímeros de línguas de suínos pela técnica da dissecação.

Para fins de denominação dos vasos descritos, utilizaram-se os termos, segundo a NOMINA ANATÔMICA VETERINÁRIA (2005): artéria lingual e artéria profunda da língua.

Para fins de quantificação, foram calculadas as frequências absolutas e relativas a partir da tabulação dos resultados.

\section{RESULTADOS E DISCUSSÃO}

Os resultados mostraram que, nos cinco animais que foram preparados para corrosão, o aporte sanguíneo para a língua foi oriundo da artéria carótida externa (Figura 1) e confirmaram igualmente a existência de um sistema de anastomose entre os antímeros linguais. Observou-se que os ramos arteriais para a língua originaram-se da artéria lingual, a qual, na região caudal ao osso hióide, tem aproximadamente dois milímetros de diâmetro. Na borda lateral do corno do osso hióide, a artéria lingual encontrava-se ventralmente ao músculo hioglosso emitindo pequenos ramos ventrais para a região hióidea, resultado também encontrado por BERNARDINO JÚNIOR et al. (2003) em suínos da linhagem Resende e por MUNIZ et al. (2006) em suínos da raça Piau. Após penetrar na musculatura intrínseca, na maioria dos casos, a artéria alcançou o tecido conjuntivo entre os músculos hioglosso e genioglosso. Os ramos arteriais para os músculos estiloglosso e genioglosso e tecido conjuntivo adjacente são vasos colaterais em números variáveis.

Embora a artéria lingual seja responsável por regiões subjacentes à língua em suínos (BERNARDINO JÚNIOR et al., 2003; MUNIZ et al., 2006), há também estudos que indicam contribuição desse vaso para irrigar os lobos cervicais do timo em fetos da linhagem Camborough 25 (LIMA et al., 2009) e em adultos (SILVA et al., 1993; MACHIDA et al., 1999; RESENDE et al., 1999).

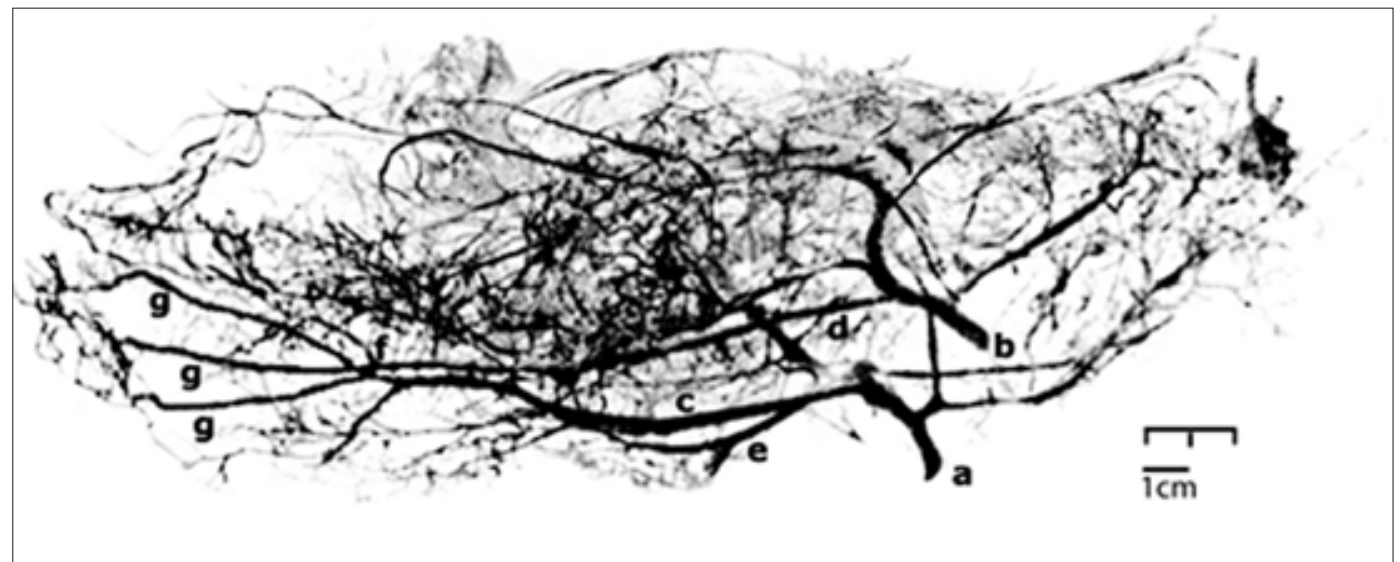

Figura 1. Fotografia do molde vascular da distribuição arterial para a cabeça de Sus scrofa domestica, em vista ventro-lateral esquerda. Evidenciam-se a artéria carótida externa no antímero esquerdo (a), artéria carótida externa no antímero direito (b); a artéria profunda da língua no antímero esquerdo (c), a artéria profunda da língua no antímero direito (d), a artéria sublingual no antímero esquerdo (e), a anastomose por inosculação entre os antímeros direito e esquerdo (f) e as artérias apicais (g).

Ao penetrar na profundidade do órgão, a artéria lingual é reconhecida na literatura como artéria profunda da língua em macaco Rhesus (Dyrud, 1944), em bovinos (GETTY, 1981), em suínos, em equinos, em carnívoros e em ovinos (SCHALLER, 1999), em suínos (BERNARDINO JÚNIOR et al., 2003; MUNIZ et al., 2006; FORTES et al., 2009), em bubalinos (MOTHEO et al., 2007) 
e em bovinos da raça Nelore (FERREIRA et al., 2009). Essa denominação é a mesma empregada pela NOMINA ANATÔMICA VETERINÁRIA (2005), tendo sido utilizada nesta pesquisa. Adotouse a denominação artéria profunda da língua a partir da porção mais rostral do terço posterior do órgão. Nesse local, a artéria correu paralela às fibras dos nervos hipoglosso e lingual, aprofundando-se no parênquima lingual e ocupando posição medial, em relação às fibras do músculo genioglosso. Essa topografia é coincidente com a artéria profunda da língua descrita pelos autores supracitados.

No terço caudal da língua, a artéria profunda da língua ascendeu rostralmente emitindo em 50\% dos casos 3 ramos dorsais primários, o primeiro desses ramos apresentou-se como um tronco de curto trajeto do qual partiu um vaso recorrente que se distribuiu no tecido conjuntivo e região do osso hióide, anastomosando-se com o ramo arterial do antímero oposto; a região suprida pelo outro vaso não foi observada por limitação da técnica. Em $46,6 \%$ das observações foram encontrados quatro ramos primários dorsais e em $3,4 \%$, cinco ramos primários dorsais para a musculatura intrínseca $\mathrm{e}$ demais tecidos componentes da língua. Esses vasos apresentaram topografia relativamente perpendicular em relação ao vaso de origem fazendo exceção o ramo mais rostral, que apresentou emergência oblíqua em sentido dorso-rostral (Tabela 1).

TABELA 1 - Representação esquemática característica dos arranjos arteriais dos ramos da artéria profunda da língua distribuídos no terço caudal dos antímeros direito e esquerdo da língua de suínos (Sus scrofa domestica, Linnaeus, 1758)

\begin{tabular}{ccc}
\hline $\begin{array}{c}\text { Variaço dos ramos dorsais } \\
\text { primários }\end{array}$ & Frequência absoluta & Frequência relativa(\%) \\
\hline & & \\
\\
\end{tabular}

Fonte: Dados da pesquisa.

Símbolos: * artéria profunda da língua

\# ramos dorsais primários da artéria profunda da língua

$>$ vaso recorrente

Em $100 \%$ dos antímeros analisados, a artéria profunda da língua, na zona limite, rostral à parte fixa do órgão, forneceu seu ramo ventral mais calibroso: a artéria sublingual, de trajeto rostral, que passa sobre a região lateral do músculo genioglosso próximo ao músculo geniohioídeo, e emitiu ramos ventrais para as estruturas componentes do assoalho da boca. RUBAY (1950) acusou a presença de vaso

similar no cavalo, EVANS (1993) no cão, DYRUD (1944) no macaco Rhesus, GETTY (1981) em ruminantes e equinos, SCHALLER (1999) em suínos e ruminantes, e DULZETTO (1968) em vertebrados genericamente. Nesta amostra pôde-se inferir que a artéria sublingual representou um ramo colateral constante responsável pelo aporte sanguíneo das estruturas miofasciais da região 
ventral do ápice lingual, com hierarquia de calibre significativa em relação ao vaso de origem. Pode-se afirmar que esse é o ramo colateral mais significativo da artéria profunda da língua de Sus scrofa doméstica (Linnaeus, 1758). Em suínos da linhagem Resende e da raça Piau, essa artéria foi observada como ramo único e ventral (BERNARDINO JÚNIOR et al., 2003) com trajeto ao longo da borda medial do músculo geniohióide (MOTHEO et al., 2006), do genioglosso, geniohióide e milohióide, além da glândula sublingual (BERNARDINO JÚNIOR, 2003). Em bubalinos a artéria sublingual foi interpretada como vaso de trajeto dorsal ao músculo geniohióide e em Bos indicus foi reconhecida como o ramo ventral de maior calibre do torus lingual (MOTHEO et al., 2007; FERREIRA et al., 2009).

Pode-se considerar, igualmente ao relato de WEISSMAN et al. (2004), que a artéria profunda da língua é o principal vaso da porção contrátil e gustatória deste órgão. O corpo e o ápice lingual são totalmente dependentes desde o vaso que é terminal a partir da emergência da artéria sublingual. Isso significa dizer que a isquemia desse vaso compromete a fisiologia da língua, órgão responsável pela apreensão de alimento, gustação e deglutição. As lesões desse vaso seguida de comprometimento fisiológico podem comprometer a estereognosia e a capacidade motora lingual (WEISSMAN et al., 2004), o que implicaria problemas na nutrição animal, muito importante em animais de alta performance genética.

Tomando como ponto de referência a emergência da artéria sublingual, são descritos a seguir os vasos dorsais do terço médio do órgão: em $46,6 \%$ das peças foram encontrados dois ramos, em $23,3 \%$ três ramos, em $23,3 \%$ quatro ramos, em $3,4 \%$ quatro ramos e em $3,4 \%$ um tronco arterial, os quatro primeiros arranjos emergindo obliquamente em direção ao dorso do órgão. Os ramos ventrais nessa região são todos de calibre fino (tabela 2).

TABELA 2 - Representação esquemática característica dos arranjos arteriais dos ramos da artéria profunda da língua e artéria sublingual distribuídos nos antímeros direito e esquerdo do terço médio da língua de suínos (Sus scrofa domestica, Linnaeus, 1758)

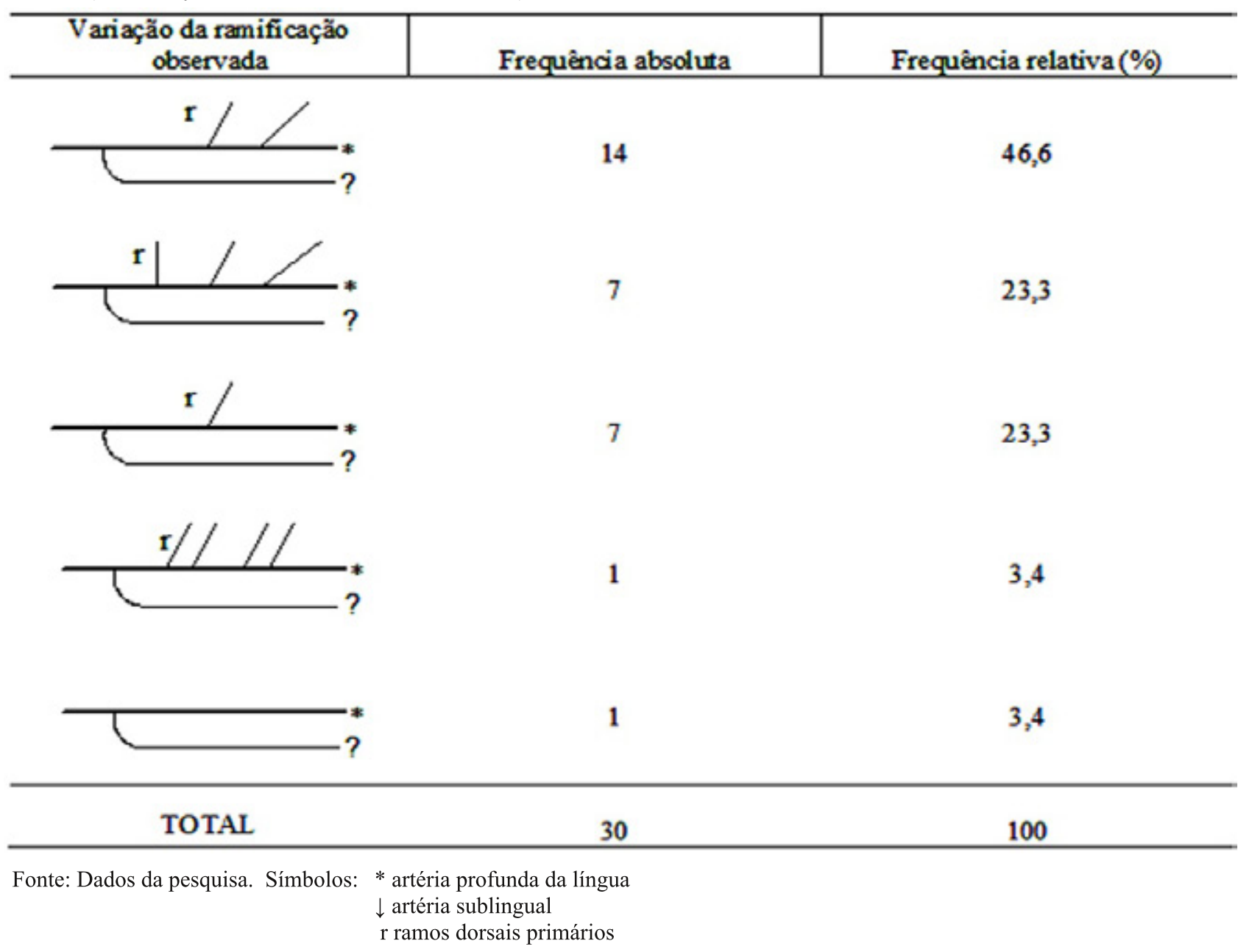


Em relação aos ramos dependentes da artéria profunda da língua é certo afirmar que depois que o vaso atinge o interior do órgão, ele transita preferencialmente entre o tecido conjuntivo das junções intermusculares; o mesmo fenômeno acontece em relação à artéria sublingual.

Os ramos dorsais e ventrais de menor calibre, representados nas tabelas 1 e 2 , tiveram suas trajetórias entre as estruturas mioconjuntivas do órgão.

Embora SCHALLER (1999) tenha esquematizado a artéria lingual em suínos como um ramo colateral da artéria carótida externa, o texto não esclarece quando esse vaso assume a condição de artéria profunda da língua, vaso igualmente ilustrado pelo autor. Considerou-se que, após cruzar o osso hióide, a artéria lingual iniciou a sua penetração intravisceral no sentido rostro-medial. Ao cruzar as fibras do músculo hioglosso, ela se encontra na intimidade do órgão podendo, a partir desse ponto, ser considerada como artéria profunda da língua.
Com relação aos ramos terminais derivados dos ramos dorsais dependentes da artéria profunda da língua, tendo como base os moldes arteriais obtidos por corrosão (10 antímeros), pode-se afirmar que esses terminaram subdividindo-se, originando capilares em formação arboriforme proximamente à mucosa lingual (Figura 2). O limite do terço médio para o terço cranial da língua representou a zona de continuidade do ramo arterial principal, presente em $100 \%$ das peças, de direção rostral que se distribuiu na musculatura intrínseca do ápice do órgão. A artéria profunda continuou e, próximo ao ápice do órgão, anastomosou-se, por inosculação, com a artéria do antímero oposto em um, dois ou três pontos, formando uma ou duas ilhas arteriais (Tabela 3). Logo após, capilarizou-se em rede no ápice lingual. No que tange a esses aspectos, não foram encontrados dados similares passíveis de comparação, embora alguns autores se refiram a anastomoses entre os antímeros em Bos indicus (FERREIRA et al., 2009).

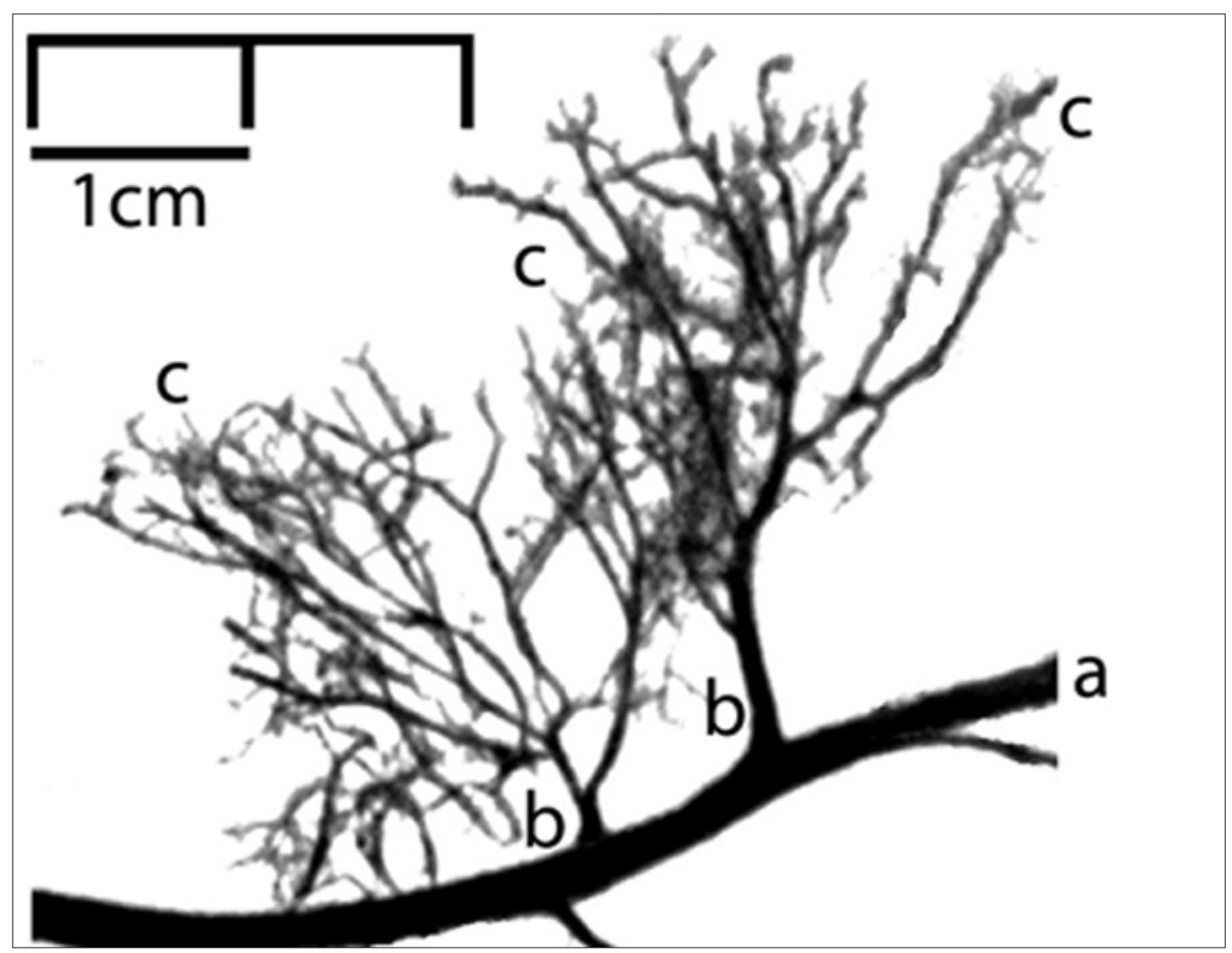

FIGURA 2. Fotografia do molde vascular da artéria profunda da língua (a) de suíno. Visualizam-se as ramificações primárias na região do dorso lingual (b) e as terminações arboriformes (c) na região da mucosa lingual. 
TABELA 3 - Representação esquemática do modo de terminação das artérias profundas da língua nos antímeros direito e esquerdo na região do ápice lingual em línguas de suínos (Sus scrofa domestica, Linnaeus, 1758)

\begin{tabular}{l|c|c}
\hline $\begin{array}{c}\text { Modos de terminaçöes } \\
\text { observados nos antimeros }\end{array}$ & Frequência absoluta & Frequência relativa(\%) \\
\hline & 14 & 46,6 \\
\hline
\end{tabular}

Fonte: Dados da pesquisa.

Siglas: $\mathrm{AD}=$ antímero direito; $\mathrm{AE}=$ antímero esquerdo.

\section{CONCLUSÕES}

A artéria profunda da língua representou o ramo de continuação rostral mais calibroso da artéria lingual. Constatou-se a presença de ramos colaterais primários dorsais linguais na região do terço lingual posterior; a artéria sublingual representa o ramo colateral mais calibroso da artéria profunda da língua; os ramos colaterais dorsais da artéria profunda da língua, em qualquer dos seus segmentos, dirigiam-se entre a massa muscular e a mucosa lingual, onde se capilarizaram; as subdivisões dos ramos dorsais da artéria sublingual e da região anterior da língua são representadas por finos vasos que irrigam os tecidos subjacentes e a mucosa do dorso ou da região ventral; a artéria profunda da língua anastomosou-se por inosculação com a artéria do antímero correspondente e, a partir dessa junção, originou-se plexo na região apical; no ápice lingual, após anastomoses entre os antímeros, ocorreu a formação de ilha arterial única ou dupla.

\section{REFERÊNCIAS}

BROWN, M. E. The occurrence of arterio-venous anastomoses in the tongue of the dog. Anatomical Record., v.69, 287-297, 1937.
DELMANN, H. D.; Brown, E. Histologia veterinária. Rio de Janeiro: Guanabara Koogan, 1982. 168p.

DYRUD, J. The external carotid artery of the rhesus monkey (Macaca mulatta). Anatomical Record, v. 9, p. 17-22, 1944.

DULZETTO, F. Anatomia comparata dei vertebrati. Paris: Edizione Caderini, 1968. p.1657-1673.

EVANS, H. E. Miller's Anatomy of the Dog. 3rd ed. Philadelphia: W. B. Saunders Company, 1993. p.608-609.

FORTES, P. M.; FERREIRA, J. R.; DIAS, A. G. C.; SILVA, R. A. Anatomia dos ramos linguais do nervo hipoglosso em Sus scrofa domesticus, L., 1758. Acta Scientiarum Biological Sciences, v. 31, n. 1, p. 97-103, 2009.

FERREIRA, J. R.; NOGUEIRA, D. J.; RODRIGUES, B. F.; ALVARENGA, B. F. Blood supply in the tongue of Nellore Bos indicus (Linnaeus, 1758). Anatomia Histologia Embryologia, v. 38, p. 103-107, 2009.

GETTY, R. Anatomia os animais domésticos. 5.ed. Rio de Janeiro: Interamericana, 1981. p. 537-538, 1228-1230.

GRANT, R. T. Observations on direct communications between arteries and veins in the rabbit's ear. Heart, v. 15, p. 281-303, 1930.

GRANT, R. T.; BLAND, E. F. Observations on arteriovenous anastomoses in human skin and in the bird's foot with special reference to the action to cold. Heart, v. 15, p. 385-411, 1931.

INTERNATIONAL COMMITEE ON VETERINARY 
GROSS ANATOMICAL NOMENCLATURE. Nomina Anatomica Veterinaria. 5. ed. Hannover, Editorial Committee, 2005.

LESBRE, F. X. Langue. In: LESBRE, F. X. Prècis d'anatomie Comparèe Dês Animaux Domestiques. Paris: J.B. Barllière et fils, 1923. p. 528-529

LIMA, E. M. M.; PAIM, T.P.; ROCHA, L.D.; BORGES, B. O.; MORAES, D. D.; CARNEIRO E SILVA, F. O.; SEVERINO, R. S.; DRUMMOND, S. S. Suprimento arterial dos lobos cervicais do timo em fetos de suínos (Sus scrofa domesticus) da linhagem Camborough 25. Biotemas, v. 22, n. 3, p. 165-169, 2009.

MACHIDA, H.; PERKINS, E.; GIACOMETTI, L. The anatomical and histochemical properties of the tongue of primates. Folia Primatologica, v. 5, n.4, p. 264-279, 1967.

MONGIARDINO, T. Manuale di anatomia descrittiva comparata degli animali domestici. Torino: Unione Tipográfico, 1905. p. 203-211.

MONTANÉ, L. ; BOURDELEE, E.; BRESSOU, C. Anatomie regionale des animaux domestiques. 9. ed. Paris: J. B. Baillière, 1949. 313p.

MOTHEO, T. F.; MACHADO, M. R. F.; SOUZA, W. M.; OLIVEIRA, F. S. Origem e ramificação do tronco linguofacial em fetos de bufalinos sem raça definida (Bubalus bubalis bubalis). Acta Scientiae Veterinariae. v. 35 , n. 2, p. 227-231, 2007).

MUNIZ, W. F.; SEVERINO, R. S.; FARIA, K. G.; MIRANDA, R. L.; ALVES, E. C. M.; CARNEIRO E SILVA, F. O.; DRUMMOND, S. S. Origens e distribuições das artérias linguais em suínos (Sus scrofa domesticus Linnaeus, 1758) da raça Piau. Bioscience Journal, v. 22, n. 3, p. 149-157, 2006.

PEREZ, N. C. Anatomia Topografica Dell Caballo. 2.ed. Barcelona: Editorial Labor, 1956. p. 89-90.

POPOFF, N. W. The digital vascular system. Archives Pathology, v.18, 295-330, 1934.

PRICHARD, M. M. L.; DANIEL, P. M. Arterio-venous anastomoses in the tong of the dog. Journal of Anatomy London, v. 87, p. 66-74, 1953.

PRICHARD, M. M. L.; DANIEL, P. M. Arterio-venous anastomoses in the tongue of the sheep and the goat.
American Journal Anatomy, v. 95, n.2, 203-225, 1954.

ROMER, A. S., PARSONS, T. S. Anatomia comparada dos vertebrados. São Paulo: Atheneu Editora, 1985. p. 265-266.

RUBAY, P. Tratado de anatomia topografica dell caballo. Madrid: Cultura Clássica e Moderna, 1950. $356 \mathrm{p}$.

SAPPEY, P. C. Système Muqueux. In: SAPPEY, P. C. Traitè d'anatomie générale. Paris: Battaille et Cie Editeurs, 1894. p.707-717.

SCHALLER, O. Sphanchnologia. In: SCHALLER, O. Nomenclatura Anatômica Veterinária Ilustrada. São Paulo: Manole, 1999. p. 140-230.

SCHWARZE, E.; SCHRÖDER, L. Compêndio de anatomia veterinária. Saragoza: Acribia, 1970. p. 3638 .

SHUMACHER, S. Über die Bedeutung der arteriovenösen anastomosen und der epitheloiden muskelzellen (Quellzellen). Zeitschrift fur MikroskopischAnatomische Forschung, v. 43, 107-130, 1908.

SILVA, F. O. C.; SEVERINO, R. S.; DRUMMOND, S. S.; CAMPOS, A. B.; CAMPOS, D. B.; REIS, F. A. C.; BENTO, L. R. T.; LIMA, E. M. M. Artérias dos lobos cervicais do timo em fetos de suínos da raça piau. Ars Veterinária, v. 19, n. 1, p. 8-12, 2003.

SONNTAG, F.C. The comparative anatomy of the tongues of the mammalia - 1. General Description of the tongue. Proceedings of Zoological Society London , v. 90 , p. $115-129,1920$.

TAYLOR, J. A. Regional and applied anatomy of the domestic animals. Edinburgh: Oliver and Boyd, 1955. p. 120-121.

WEICHERT, C. K. Lengua. In: WEICHERT, C. K. Elementos de Anatomia de Los Cordados. 2.ed. Madrid: McGrawhill Book Company INC., 1966. p. 119121.

WEISSMAN, R; WILLEMAN A.; BERNARDI, F. H. Necrose da lingual causada por trauma - relato de caso. Revista Portuguesa de Estomatologia, Medicina Dentária e Cirurgia Maxilofacial, v. 45, n. 2, p. 79-84, 2004. 